\title{
Photo-Induced Click Chemistry for DNA Surface Structuring via Direct Laser Writing
}

\author{
Antonina Kerbs, Patrick Mueller, Michael Kaupp, Ishtiaq Ahmed, Alexander S. Quick, Doris Abt, Martin \\ Wegener, ${ }^{[\mathrm{d}]}$ Christof M. Niemeyer, ${ }^{[\mathrm{c}]}$ Christopher Barner-Kowollik ${ }^{*[b]}$ and Ljiljana Fruk ${ }^{*[a]}$
}

\begin{abstract}
Oligonucleotides containing photo-caged dienes were prepared and shown to react quantitatively in a light-induced Diels-Alder cycloaddition with functional maleimides in aqueous solution within minutes. Due to its high yield and fast rate, the reaction was exploited for DNA surface patterning with submicrometer resolution employing direct laser writing (DLW). Functional DNA arrays were written via direct laser writing (DLW) in variable patterns, which were further encoded with fluorophores and proteins through DNA directed immobilisation. This mild and efficient light driven platform technology holds promise for fabrication of complex bioarrays with sub-micron resolution.
\end{abstract}

Short DNA strands containing functional groups, which can selectively bind to variable surfaces have found numerous applications as structural and functional elements in the design of biological assays, ${ }^{[1]}$ biosensors, ${ }^{[2]}$ nanomotors ${ }^{[3]}$ and optoelectronic devices. ${ }^{[4]}$ To enable binding of either one or several different DNA sequences, a number of structuring techniques such as photo and dip-pen lithography, ${ }^{[5]}$ nanografting, ${ }^{[6]}$ nano-pipetting ${ }^{[7]}$ or micro-contact printing ${ }^{[8]}$ have been developed in the past decades for DNA surface structuring with increased resolution. However, these methods are not only time consuming, but lack either in multiplexing ability or pattern variety (or both), and are often limited by the nature of the employed surface. To overcome the drawbacks of existing DNA structuring methods and enable one-step fabrication of DNA patterns with sub-micrometer resolution features, we here, for the first time, employ direct laser writing (DLW) and photo-

[a] Dr. A. Kerbs, Dr. L. Fruk

Department of Chemical Engineering and Biotechnology University of Cambridge, New Museums Site, Pembroke Street, Cambridge, CB2 3RA (UK), E-mail: If389@cam.ac.uk

[b] Dr. M. Kaupp, Dr. A. S. Quick, D. Abt, Prof. C. Barner-Kowollik Preparative Macromolecular Chemistry, Institute for Technical and Polymer Chemistry, Karlsruhe Institute of Technology (KIT), Engesserstrasse 18, 76131 Karlsruhe (Germany) and Institute for Biological Interfaces-3, Karlsruhe Institute of Technology (KIT), Hermann-von-Helmholtz-Platz 1, 76344 EggensteinLeopoldshafen (Germany), E-Mail: christopher.barnerkowollik@kit.edu

[c] Dr. I. Ahmed, Prof. C. M. Niemeyer Institute for Biological Interfaces-1, Karlsruhe Institute of Technology (KIT), Hermann-von-Helmholtz-Platz 1, 76344 EggensteinLeopoldshafen (Germany)

[d] P. Mueller, Prof. M. Wegener, Institute of Nanotechnology, Karlsruhe Institute of Technology (KIT), Hermann-von-HelmholtzPlatz 1, 76344 Eggenstein-Leopoldshafen (Germany)

Supporting information for this article is given via a link at the end of the document. induced click chemistry to covalently anchor single-stranded DNA (ssDNA) to glass surfaces. The orthogonal light-induced Diels-Alder cycloaddition between photo-caged dienes and maleimide groups on the surface has previously been exploited in combination with DLW to attach peptides, ${ }^{[9]}$ biotin and benzylguanine to glass slides for selective immobilization of streptavidin and SNAP tag labeled fluorescent proteins, respectively. ${ }^{[10]}$ The ligation reaction proceeds under UV irradiation, yet the use of two-photon laser writing strategy makes our approach suitable for immobilization of a range of UV sensitive biomolecules such as DNA. The multiphoton-induced Diels-Alder cycloaddition ${ }^{[11]}$ has already been successfully employed by us for the fabrication of woodpile photonic crystals, ${ }^{[12]}$ for the coating of pre-formed 3D polymeric microstructures, ${ }^{[13]}$ immobilization of functionalized gold nanoparticles ${ }^{[14]}$ and for the wavelength selective polymer network formation. ${ }^{[15]}$

The methodology based on DLW presented in the current study allows for the one-step fabrication of pre-programmed 2D DNA patterns on glass surfaces with sub-micrometer resolution. Once written, such patterns can further be encoded with various species such as fluorophores or proteins through DNA directed immobilization. ${ }^{[16]}$ To achieve the surface patterning, photoactive oligonucleotides containing the 2-formyl-3-methylphenoxy derivative 1 (FMP) moiety, which isomerizes to a reactive dienol (photoenol) under irradiation, ${ }^{[17]}$ were synthesized by amide coupling of FMP-NHS ester 2 and amino-terminated DNA $\left(\mathrm{NH}_{2}-\mathrm{DNA}\right)$ immobilized on controlled porous glass (CPG) supports (Scheme 1).

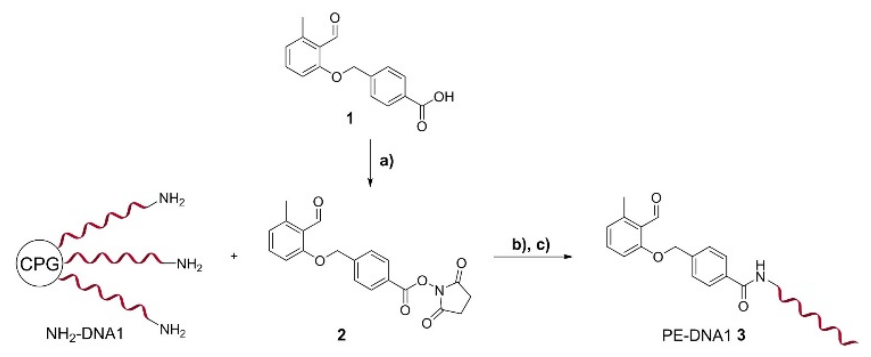

Scheme 1. Synthesis of PE-NHS ester 2 and amide coupling to $\mathrm{NH}_{2}-\mathrm{DNA} 1$ to afford photoactive PE-DNA1 3. (a) NHS, DCC, THF, $0{ }^{\circ} \mathrm{C}-\mathrm{RT}, 16 \mathrm{~h}$, quantitative; (b) DIPEA, DMF, $40{ }^{\circ} \mathrm{C}, 2 \mathrm{~h}$; (c) $25 \% \mathrm{NH}_{3}, 55^{\circ} \mathrm{C}, 5 \mathrm{~h}$.

Two photo-active oligonucleotides PE-DNA1 3 and PE-DNA2 6 were prepared in $35 \%$ and $40 \%$ yields respectively, purified by reversed phase HPLC (SI, Figure S3, S4,) and their identity confirmed by MALDI-TOF mass spectrometry (SI, Table S2). To enable direct laser writing and efficient patterning, the photo- 
A

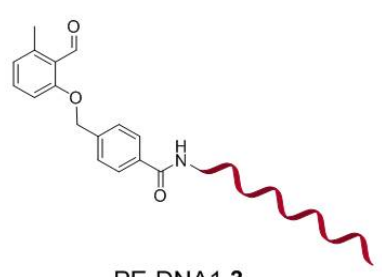

PE-DNA1 3
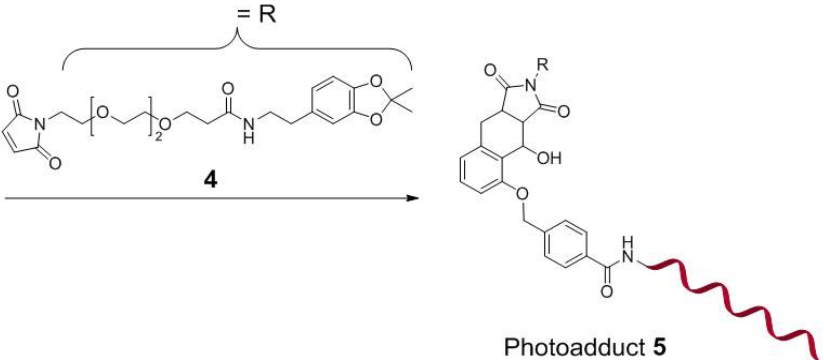

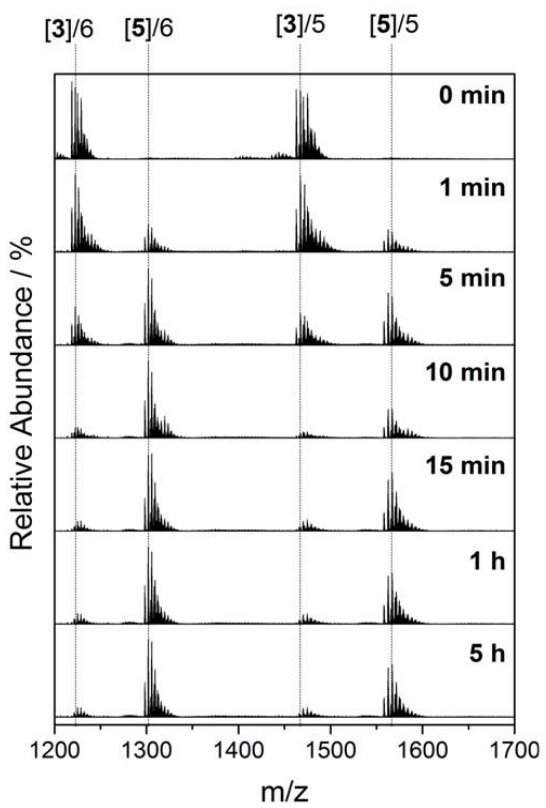

$\mathrm{C}$

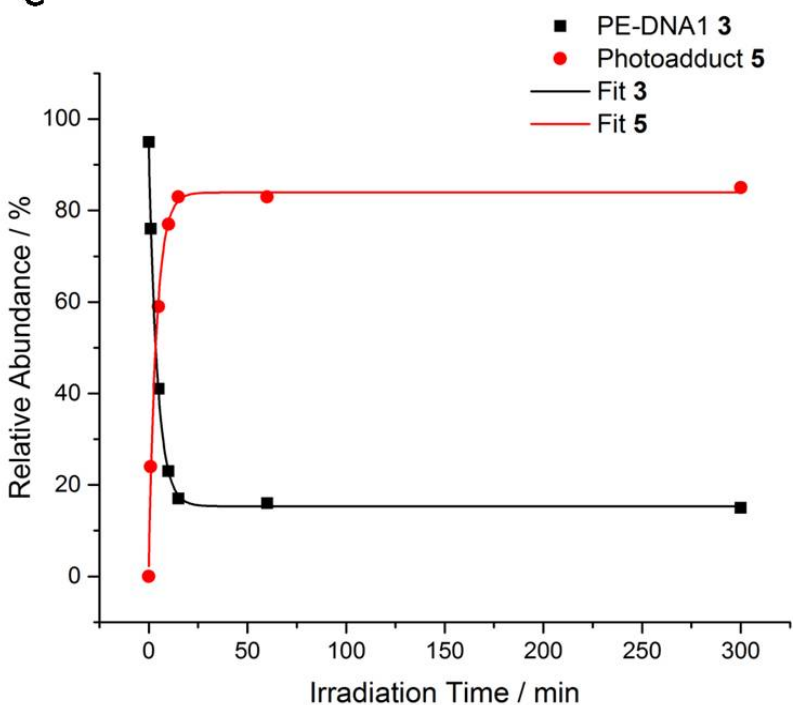

Figure 1. Kinetic study of the photoreaction between PE-DNA1 3 and Do-TEG-Mal 4 under irradiation with an UV-lamp (Arimed B6, $\lambda$ max $=320 \mathrm{~nm} \pm 30 \mathrm{~nm})$. A: Scheme of the photoreaction between PE-DNA1 3 and Do-TEG-Mal 4; 1.00 eq. PE-DNA1 3, 15.0 eq. Do-TEG-Mal 4, water/DMSO solution (3/2. v/v). B: High resolution ESI mass spectra, recorded after $0,1,5,10,15 \mathrm{~min}, 1 \mathrm{~h}$ and $5 \mathrm{~h}$ irradiation, depict 5-fold and 6-fold charged ions of PE-DNA1 3 and photoadduct 5. C: Kinetic plots derived from integration of the corresponding mass spectra shown in $\mathrm{B}$.

induced reaction needs to be relatively fast and proceed without the formation of side products. Therefore, we initially explored the reaction kinetics of PE-DNA1 3 under light irradiation with model dienophile dopamine-triethylene glycol-maleimide (Do-TEG-Mal) 4. The kinetics of the reaction were followed by high resolution electrospray ionization mass spectrometry (ESIMS) at preset irradiation times (Figure 1A). From the obtained mass spectra it can be seen that the 5-fold and the 6-fold charged ions of PE-DNA1 3 at $\mathrm{m} / \mathrm{z}=1467.2625$ and $\mathrm{m} / \mathrm{z}=1222.5521$ disappear over time, while there is an increase of the relative abundances of 5 -fold and 6 -fold charged ions of the cycloadduct 5 at $\mathrm{m} / \mathrm{z}=1567.0983$ and $\mathrm{m} / \mathrm{z}=1301.9206$ (Figure 1B).

The percentage integral values of the recorded signals from the mass spectra were plotted as a function of time indicating excellent agreement of the experimental data points with an exponential fit, suggesting pseudo first-order kinetics (Figure 1C) As seen from the mass spectra, the quantitative conversion is achieved after 10 minutes (Figure 1B). Detailed analysis of the isotopic pattern of the most abundant isotopes and the comparison to the simulated isotopic pattern (SI, Figure S 6) additionally confirms the successful generation of the desired cycloadduct 5 . It should be noted that approximately $16 \%$ of PE-DNA1 3 remained in the reaction mixture after irradiation and the conversion could not be increased by prolonged irradiation time or by using high excess of dienophile 4 (up to 100 eq., SI, Figure $S 5$ ). This may be explained by an unfavorable PE-DNA1 3 conformation, which might hinder the accessibility of FMP 1 moiety.

After successful assessment of the model reaction, DLW was used for ssDNA structuring onto glass surface modified to contain maleimide functional groups ( $\mathrm{SI}$, experimental details and Scheme S1). A custom designed DLW setup was employed as we use a laser tuned to $700 \mathrm{~nm}$ center wavelength for targeted two-photon excitation, ensuring the efficient absorption by PE-DNA1 3, which is required for the photo-induced cycloaddition. In order to achieve efficient fabrication of DNA patterns, the writing conditions were first optimized by conducting a range of dose and resolution tests using $10 \mu \mathrm{M}$ PE-DNA1 3 dissolved in a mixture of $0.5 \times \mathrm{SSC}(\mathrm{pH} 7.5)$ and 
DMSO $(3 / 2, v / v)$ at the writing speed of $100 \mu \mathrm{m} \cdot \mathrm{s}^{-1}$. Dose test patterns allow for the determination of the optimal laser power range and correct glass interface detection (SI, experimental details and Figure S 7). In addition, further test patterns aid in determining the maximum achievable resolution for the given DLW system. Line segments with varying line distances ranging from $1 \mu \mathrm{m}$ to $0.4 \mu \mathrm{m}$ (Figure $2 \mathrm{~B}$ ) and laser power ranging from $0.4 \mathrm{~mW}$ to $24 \mathrm{~mW}$ (Figure 2B, left image, power increases from left to right), were fabricated on the maleimide functionalized glass surface using PE-DNA1 3. To visualize the written ssDNA pattern, the surfaces were incubated with a fluorophore labeled complementary oligonucleotide Cy3-cDNA1 and analyzed by fluorescence microscopy (Figure 2B).
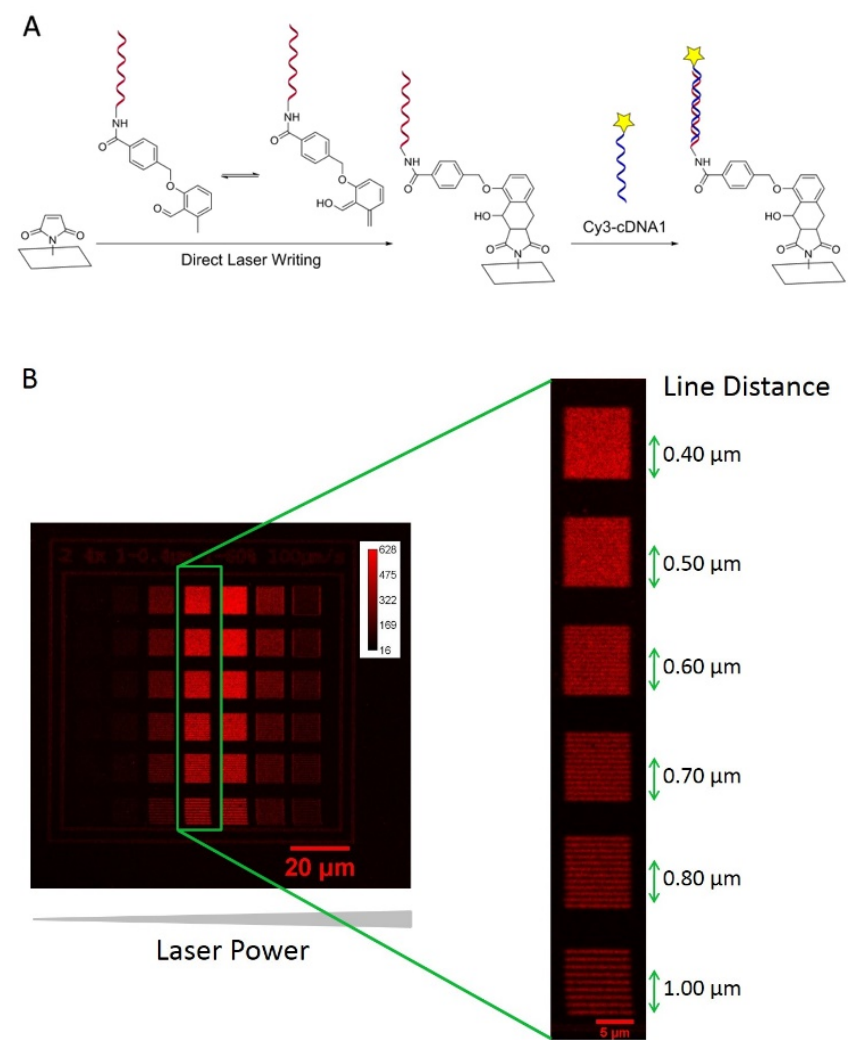

Figure 2. A: Photoenolisation of PE-DNA1 3, induced by laser light absorption, and [4+2] cycloaddition with the maleimide moiety bound to the surface and subsequent hybridisation with Cy3-cDNA1. B: Fluorescence images of Cy3-cDNA1 hybridized 2D line array pattern of DNA1 written by DLW using 4-fold scanning repetition and a speed of $100 \mu \mathrm{m} \mathrm{s}^{-1}$. The laser power was increased from left to the right: $0.4 \mathrm{~mW}, 0.8 \mathrm{~mW}, 2 \mathrm{~mW}, 4 \mathrm{~mW}, 8 \mathrm{~mW}, 16 \mathrm{~mW}$ and $24 \mathrm{~mW}$. Zoomed section showing different line patterns written using $4 \mathrm{~mW}$ laser power with numbers indicating the distances between the line segments.

As it can be seen from Figure $2 \mathrm{~B}$, strong fluorescent signals were obtained from regions written using 4 and $8 \mathrm{~mW}$ laser powers. The absence of the fluorescent signals in the areas without immobilised DNA demonstrated the high precision of DLW and the high specificity of DNA-directed immobilisation through double strand formation. When patterns were written using low and high laser power, weak fluorescence is observed indicating either low activation of PE-DNA1 3 (low power), or its destruction (high power). The magnification of the segment written with $4 \mathrm{~mW}$ laser power (Figure 2B, right) shows well defined lines with the width of less than $500 \mathrm{~nm}$ at line distances as low as $600 \mathrm{~nm}$ (as determined from the fluorescence intensity profile across the lines, SI, Figure S8). To the best of our knowledge, this result represents the highest resolution achieved by photolithographic immobilization of pre-synthesized oligonucleotides. ${ }^{[18]}$

After successful immobilisation of one oligonucleotide sequence, we assessed the potential of the employed patterning technique for the fabrication of multi-sequence DNA patterns. As a proof of concept, DNA patterns were prepared using two sequences, i.e. PE-DNA1 3 and PE-DNA2 6 modified with photo-active groups. The fabrication was performed in three writing steps. First, a $100 \times 100 \mu \mathrm{m}^{2}$ polymer frame (Figure S9) was written using a photoresist (tetra-FMP and maleimide containing methacrylate derivative) solution in $\mathrm{y}$-butyrolacton/acetophenone mixture. ${ }^{[12]}$ The fabricated polymer frame is visible with a CCD camera and serves as a reference point for the control of the laser position. In the next step, line segments (line distances: $8.00 \mu \mathrm{m}, 6.00 \mu \mathrm{m}$ and $4.00 \mu \mathrm{m}$, Figure 3) were written using PE-DNA1 3.

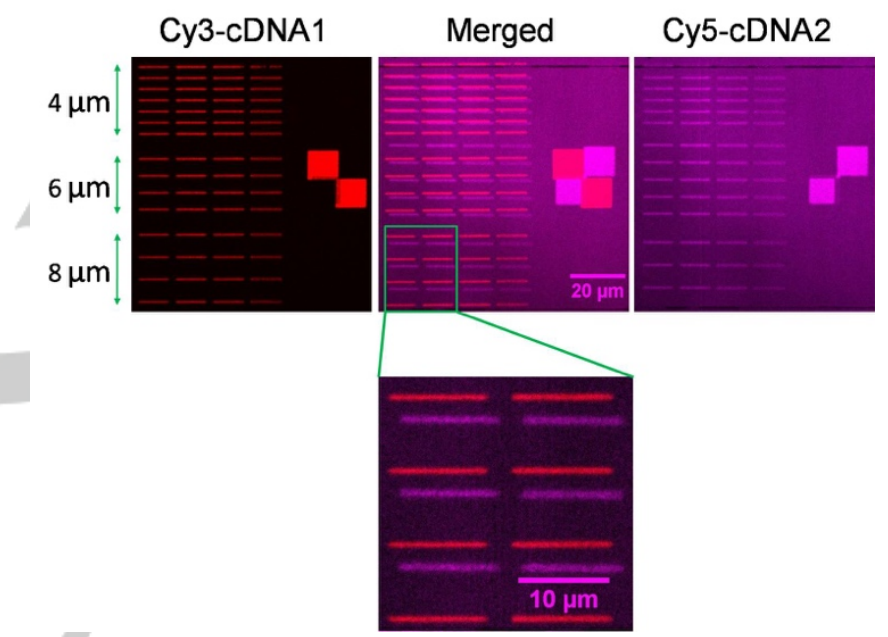

Figure 3. Top: fluorescence images of the two DNA sequence patterns for Cy3 (left, red $\lambda_{\mathrm{ex}}=550 \mathrm{~nm}, \lambda_{\mathrm{em}}=570 \mathrm{~nm}$ ), Cy5 (right, violet $\lambda_{\mathrm{ex}}=649 \mathrm{~nm}$, $\lambda_{\mathrm{em}}=666 \mathrm{~nm}$ ) and merged signals (middle). Bottom: magnification of the immobilised DNA lines of the merged image (red, DNA1-cDNA1, violet DNA2cDNA2).

In addition, a $2 \times 2$ checkerboard pattern was written on the right hand side of the frame. In the final step, the second sequence PE-DNA2 6 was written in between the lines and in a square pattern to complete the checkerboard. Once two sequence patterns were written, hybridization with complementary fluorophore labeled DNA sequences, Cy3-cDNA1 and Cy5-cDNA2 was performed to visualize the patterns (Figure 3). Strong and clearly distinguishable fluorescent signals could be detected for both sequences. Due to the limitations of the employed piezo stage used together with the CCD camera, for the localization of the start position to enable writing of the second DNA sequence, the line pattern is slightly shifted to the right. However, the high efficiency of the DNA hybridization and obtained well-resolved patterns confirm the applicability of the light-induced cycloaddition and DLW for the fabrication of DNA patterns, which can be readjusted to the needs of a particular application.

In order to test the plasticity of the DLW DNA pattern, in particular its ability to be used for immobilisation of various nonnucleic acid species, we exposed the surface to the protein 
streptavidin (STV) modified with cDNA1 ${ }^{[16 a, 16 c]}$ The STV-cDNA1 conjugate was hybridized to $2 \times 2\left(45 \times 45 \mu \mathrm{m}^{2}\right)$ checkerboard pattern containing both DNA1 and DNA2 sequences $(100 \mathrm{nM}$ STV-cDNA1 solution in PBS buffer at ambient temperature for $2 \mathrm{~h}$ ). To evidence that STV-cDNA1 is bound specifically to the DNA1 containing pattern, fluorophore labeled biotin (Atto550biotin, $300 \mathrm{nM}$ ) was added to the array and incubated for $2 \mathrm{~h}$. As it can be seen in Figure 4B high fluorescence intensity of Atto550 can be observed in regions with patterned DNA1, indicating both that STV protein can be selectively and specifically immobilized and that it remains in its functional, biotin-binding conformation.

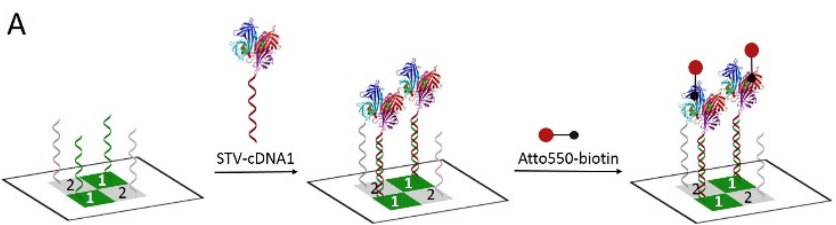

B

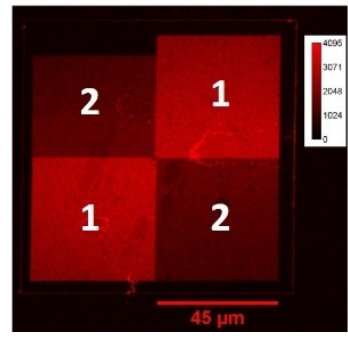

C

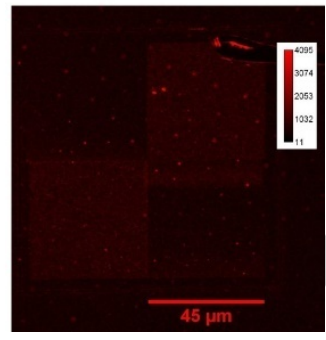

Figure 4. A: Scheme of DNA directed immobilisation of STV-cDNA1 conjugate on two-sequence DNA array and subsequent Atto550-biotin binding. B: Fluorescence images of Atto550-biotin bound to two sequence pattern containing immobilised STV-cDNA1 (squares labeled 1). C: Fluorescence images of Atto550-biotin on a STV free surface. Atto550: $\lambda_{\mathrm{ex}}=554 \mathrm{~nm}$, $\lambda_{\mathrm{em}}=576 \mathrm{~nm}$.

Low fluorescence intensity can be observed in the control regions containing DNA2 only (Figure 4B, region 2) and DNA1 without STV (Figure 4C), but not in the surrounding DNA free area, which we attribute to the weak electrostatic interaction of the cationic fluorophore Atto550 and the anionic oligonucleotide backbone. This issue can be resolved by the use of other fluorophores and more intense washing steps.

In conclusion, we have for the first time demonstrated that photo-induced Diels-Alder click chemistry combined with direct laser writing can be used for the precise patterning of DNA on the maleimide-functionalized surfaces. Owing to its remarkable resolution and the variety of patterns (including 3D) which can be fabricated, this methodology should be applicable in numerous areas of bionanotechnology and surface structuring, for instance for immobilisation of seed cells through patterns of cell receptors. We thus believe that our method holds a great potential for the future application in cell and tissue growth, and preparation of bio-inspired materials, which is the topic of our ongoing studies.

\section{Acknowledgements}

This work was supported by the Helmholtz programme Biolnterfaces in Technology and Medicine (BIFTM). C.B.-K. acknowledges continued funding from the Karlsruhe Institute of Technology (KIT) in the context of the BIFTM and STN programs. We acknowledge Prof. Martin Bastmeyer (KIT) for providing the laser-scanning fluorescence microscope. P. M and M.W.acknowledge support by the Helmholtz program Science and Technology of Nanosystems (STN) and by the Karlsruhe School of Optics \& Photonics (KSOP).

Keywords: Light-active Oligonucleotides - DNA Directed Immobilisation • Direct Laser Writing (DLW) • DNA Microarrays

[1] L. Ma, M. Su, T. Li, Z. Wang, Analyst 2013, 138, 1048-1052.

[2] a) E. Palecek, M. Bartosik, Chem. Rev. 2012, 112, 3427-3481; b) K. M. Abu-Salah, M. M. Zourob, F. Mouffouk, S. A. Alrokayan, M. A. Alaamery, A. A. Ansari, Sensors 2015, 15, 14539-14568.

[3] J. Cheng, S. Sreelatha, I. Y. Loh, M. Liu, Z. Wang, Methods 2014, 67, 227-233.

[4] S. M. Yazdi, Y. Yuan, J. Ma, H. Zhao, O. Milenkovic, Sci. Rep. 2015, 5, 14138.

[5] a) M. C. Pirrung, Angew. Chem. 2002, 114, 1326-1341; Angew. Chem. Int. Ed. 2002, 41, 1276-1289; b) L. M. Demers, D. S. Ginger, S. J. Park, Z. Li, S. W. Chung, C. A. Mirkin, Science 2002, 296, 1836-1838.

G. Y. Liu, N. A. Amro, Proc. Natl. Acad. Sci. 2002, 99, 5165-5170.

[7] A. Bruckbauer, D. Zhou, L. Ying, Y. E. Korchev, C. Abell, D.

[7] A. Bruckbauer, D. Zhou, L. Ying, Y. E. Korchev, C. Abell, D. S. A. Lange, V. Benes, D. P. Kern, J. K. Horber, A. Bernard, Anal. Chem. 2004, 76, 1641-1647.

[9] T. Pauloehrl, G. Delaittre, V. Winkler, A. Welle, M. Bruns, H. G. Borner, A. M. Greiner, M. Bastmeyer, C. Barner-Kowollik, Angew. Chem. 2012, 124, 1096-1099; Angew. Chem. Int. Ed. 2012, 51, 1071-1074.

[10] B. Richter, T. Pauloehrl, J. Kaschke, D. Fichtner, J. Fischer, A. M. Greiner, D. Wedlich, M. Wegener, G. Delaittre, C. Barner-Kowollik, M. Bastmeyer, Adv. Mater. 2013, 25, 6117-6122.

[11] G. Delaittre, A. S. Goldmann, J. O. Mueller, C. Barner-Kowollik, Angew. Chem. 2015, 127, 11548-11564; Angew. Chem. Int. Ed. 2015, 54, 11388-11403.

[12] A. S. Quick, H. Rothfuss, A. Welle, B. Richter, J. Fischer, M. Wegener, C. Barner-Kowollik, Adv. Funct. Mater. 2014, 24, 35713580 .

[13] E. Blasco, B. Yameen, A. S. Quick, P. Krolla-Sidenstein, A. Welle, M. Wegener, C. Barner-Kowollik, Macromolecules 2015, 48, 87188728.

[14] L. Stolzer, A. S. Quick, D. Abt, A. Welle, D. Naumenko, M. Lazzarino, M. Wegener, C. Barner-Kowollik, L. Fruk, Chem. Commun. 2015, 51, 3363-3366.

[15] M. Kaupp, K. Hiltebrandt, V. Trouillet, P. Mueller, A. S. Quick, M. Wegener, C. Barner-Kowollik, Chem. Commun. 2016, 52, 19751978.

[16] a) L. Fruk, J. Muller, G. Weber, A. Narvaez, E. Dominguez, C. M. Niemeyer, Chem. Eur. J. 2007, 13, 5223-5231; b) H. Schroeder, B. Ellinger, C. F. Becker, H. Waldmann, C. M. Niemeyer, Angew. Chem. 2007, 119, 4258-4261; Angew. Chem. Int. Ed. 2007, 46, 4180-4183; c) C. M. Niemeyer, L. Boldt, B. Ceyhan, D. Blohm, Anal. Biochem. 1999, 268, 54-63.

[17] J. L. Segura, N. Martin, Chem. Rev. 1999, 99, 3199-3246.

[18] a) F. Huang, H. Xu, W. Tan, H. Liang, ACS Nano 2014, 8, 68496855 ; b) J. Escorihuela, M. J. Banuls, R. Puchades, A. Maquieira, Bioconjugate Chem. 2012, 23, 2121-2128. 


\section{COMMUNICATION}

A Laser Pen for DNA Writing: Photoactive oligonucleotides were patterned onto maleimide coated glass surface using direct laser writing (DLW) and light-induced Diels-Alder cycloaddition. The patterns with sub-micrometer resolution can further be used for the immobilisation of various molecular species such as fluorophores and proteins.
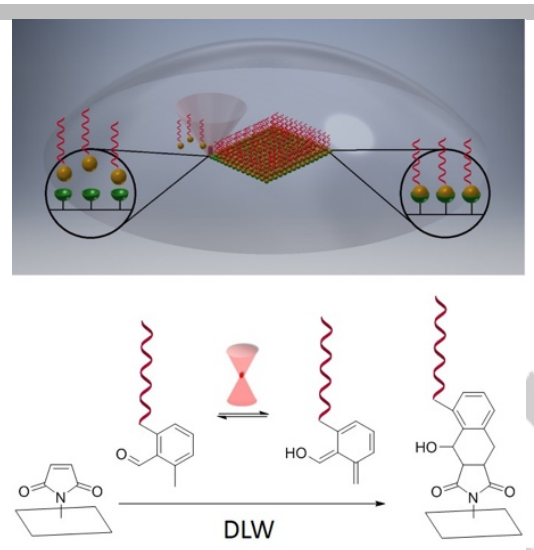

Antonina Kerbs, Patrick Mueller,

Michael Kaupp, Ishtiaq Ahmed,

Alexander S. Quick, Doris Abt, Martin Wegener, Christof M. Niemeyer, Christopher Barner-Kowollik* and Ljiljana Fruk ${ }^{*}$

Page No. - Page No.

Photo-Induced Click Chemistry for DNA Surface Structuring via Direct Laser Writing 